\title{
A velocidade de processamento e as dificuldades de aprendizagem na aritmética
}

\section{The processing speed and the arithmetic's learning difficulties}

\section{La velocidad de procesamiento y las dificultades de aprendizaje en la aritmética}

\section{Luciana Vellinho Corso*}

Universidade Federal do Rio Grande do Sul - UFRGS, Porto Alegre, Rio Grande do Sul, Brasil

\author{
Beatriz Vargas Dorneles** \\ Universidade Federal do Rio Grande do Sul - UFRGS, Porto Alegre, Rio Grande do \\ Sul, Brasil
}

\begin{abstract}
RESUMO
Estudos em velocidade de processamento, uma das habilidades cognitivas subjacentes à aprendizagem da aritmética, têm despertado o interesse de pesquisadores nas áreas de educação matemática, psicologia cognitiva e neuropsicologia. A literatura indica que uma baixa velocidade de processamento é uma das características dos alunos que apresentam dificuldades de aprendizagem na aritmética. Este artigo apresenta os resultados de pesquisa, ainda pouco conclusivos, que relacionam a velocidade de processamento e as dificuldades na aritmética. Discute a relação entre a velocidade de processamento e a memória de trabalho, outra habilidade cognitiva fundamental para a aprendizagem da matemática, e destaca a perspectiva de complementaridade entre estas duas funções. Por fim, o artigo relaciona a velocidade de processamento e a fluência em fatos aritméticos, e dá ênfase à hipótese de que uma baixa velocidade de processamento contribui para sobrecarregar a memória de trabalho acarretando dificuldades para a recuperação de fatos da memória de longo prazo. Destaca-se a necessidade de mais pesquisa para que se possa melhor definir o papel da velocidade de processamento na aprendizagem da aritmética e, assim, avançar na intervenção preventiva e corretiva das dificuldades nesta área.
\end{abstract}

Palavras-chave: Velocidade de processamento, Memória de trabalho, Dificuldades de aprendizagem na aritmética.

\begin{abstract}
Studies in processing speed, one of the cognitive abilities underlying arithmetic learning, have attracted the interest of researchers in mathematics education, cognitive psychology and neuropsychology. The literature indicates that a low processing speed is one of the characteristics of students who have learning difficulties in arithmetic. This article presents research results, still not conclusive, linking processing speed to difficulties
\end{abstract}


in arithmetic. It discusses the relationship between processing speed and working memory, another key cognitive skill for learning mathematics, and highlights the complementary perspective between these two functions. Finally, the article relates processing speed and the fluency in arithmetic fact retrieval, and emphasizes the hypothesis that a low processing speed contributes to overload working memory, causing difficulties for retrieving facts from long term memory. The article highlights the need for more research in order to better define the role that processing speed plays in learning arithmetic, and thus, be able to advance in the preventive and corrective intervention processes in this area.

Keywords: Processing speed, Working memory, Arithmetic difficulties.

\section{RESUMEN}

Los estudios realizados en la velocidad de procesamiento, una de las habilidades cognitivas subyacentes a aprendizaje de la aritmética, han atraído el interés de los investigadores en educación matemática, la psicología cognitiva y la neuropsicología. La literatura indica que una baja velocidad de procesamiento es una de las características de los estudiantes que tienen dificultades de aprendizaje artimética. En este artículo se presentan los resultados de la investigación, poco concluyente de que se refieren a la velocidad de procesamiento y las dificultades para la aritmética. Discute la relación entre la velocidad de procesamiento y la memoria de trabajo, otra habilidad cognitiva fundamental para el aprendizaje de matemáticas, y pone de relieve la perspectiva de complementariedad entre estas dos funciones. Por último, el artículo relaciona la velocidad de procesamiento y la fluidez en hechos aritméticos, y hace hincapié en la hipótesis de que una baja velocidad de procesamiento contribuye a la sobrecarga de la memoria de trabajo que causa dificultades para la recuperación de datos desde la memoria a largo plazo. Destaca la necesidad de más investigación para poder definir mejor el papel de la velocidad de procesamiento en el aprendizaje de la aritmética y de este modo avanzar en la intervención preventiva y correctiva de las dificultades en esta área.

Palabras clave: Velocidad de procesamiento, Memoria de trabajo, Dificultad de aprendizaje en la aritmética.

\section{Introdução}

Pesquisas dedicadas ao estudo dos aspectos cognitivos, subjacentes à aprendizagem em diferentes domínios, têm dado papel de destaque à velocidade de processamento por ser uma das habilidades cognitivas fundamentais que apoia a aprendizagem da matemática. A velocidade de processamento refere-se à eficiência com que tarefas cognitivas simples são executadas (Geary, Hord, \& Hamson, 1999).

Este artigo apresenta resultados de pesquisas que relacionam a velocidade de processamento e as dificuldades na matemática, em particular, no cálculo. Como veremos, os estudos nesta área são ainda pouco conclusivos, porém nos fornecem pistas de que para avançarmos nas áreas de prevenção e intervenção das dificuldades nesta área, a velocidade de processamento precisa ser conhecida mais a fundo. $O$ artigo discute também a relação entre a velocidade 
de processamento e a memória de trabalho, outra habilidade cognitiva fundamental para a aprendizagem da matemática. Por fim, ao abordarmos a relação entre a velocidade de processamento e a fluência em fatos aritméticos, destacamos a hipótese de que uma baixa velocidade de processamento contribui para sobrecarregar a memória de trabalho que, por sua vez, impossibilita as associações entre problema e resposta na memória de longo prazo, acarretando dificuldades para a recuperação de fatos básicos da memória.

\section{A Velocidade de processamento e as dificuldades de aprendizagem na aritmética}

A literatura indica que a velocidade de processamento encontra-se prejudicada nos alunos que apresentam dificuldade de aprendizagem na matemática (DM). Tais alunos processam a informação mais lentamente do que seus pares sem dificuldades (Bull \& Johnston, 1997; Swanson \& Sachse-Lee, 2001; Murphy, Mazzocco, Hanich, \& Early, 2007). Os trabalhos de Geary, Brown e Samaranayake (1991) e Geary, et al. (1999) evidenciaram que os alunos com DM são mais lentos para realizar as estratégias de contagem do que seus colegas com desenvolvimento típico. Orrantia, Martinez, Morán, \& Fernández (2002) observaram que, em termos globais, os alunos com DM são mais lentos que seus iguais sem dificuldades, não somente nos tempos totais de resposta, mas também quando ambos os grupos utilizam os mesmos procedimentos. Os resultados mostram que 0 processamento numérico, especialmente a codificação de dígitos, é substancialmente mais lento nestes alunos.

Em um estudo longitudinal desenvolvido com crianças de 5 a 7 anos, Sasanguie, Van Den Bussche, \& Reynvoet (2012) constataram que duas tarefas de processamento numérico são bons preditores de desempenho aritmético, uma em maior e outra em menor extensão. A proposta com resultado mais significativo foi a que compreendia a comparação de magnitude entre matrizes de pontos (estimar se uma quantidade de pontos é maior ou menor do que um número pré estabelecido). A tarefa que avaliava a velocidade para processar magnitude entre numerais arábicos (qual o maior numeral entre dois) foi a que pareceu exercer menor influência no desempenho matemático posterior dos alunos.

Vanbinst, Ghesquiere e De Smedt (2012) investigaram medidas de processamento numérico básico que estão relacionadas com a aritmética elementar e as estratégias utilizadas para realizar estes cálculos. Os autores observaram que o processamento de magnitude numérica dos alunos, medido por tarefas de comparação de dígitos arábicos, está relacionado com a competência aritmética: Os alunos com melhor acesso à magnitude numérica de dígitos arábicos 
recuperam mais fatos da memória e são mais rápidos na execução de várias estratégias aritméticas.

Encontramos na literatura tarefas diversas utilizadas como medidas de velocidade de processamento, entre elas as tarefas de velocidade de acesso que avaliam a velocidade com que as informações fonológicas da memória de longo prazo são acessadas (Hecht, Torgesen, Wagner, \& Rashotte, 2001). A Tabela 1 mostra exemplos de tarefas utilizadas para avaliar a velocidade de processamento. 
Tabela 1

Exemplos de medidas de velocidade de processamento

\begin{tabular}{ll}
\hline Pesquisadores & Descrição da tarefa \\
\hline Andersson & Números Idênticos: A tarefa compreende uma folha de papel \\
(2008) & com 30 fileiras de 7 digitos. Em cada fileira há dois digitos \\
& $\begin{array}{l}\text { idênticos e o aluno deve encontrar os pares o mais rápido } \\
\text { possivel. O tempo levado para completar a tarefa é } \\
\text { cronometrado. }\end{array}$
\end{tabular}

Fuchs, Fletcher Números Idênticos: A tarefa consiste de 60 fileiras de seis \& Lambert dígitos em que o aluno deve circular dois dígitos iguais em cada (2008). fileira. É dado um tempo de 3 minutos para completar a tarefa.

Hecht et al., Nomeação de dígitos: A tarefa compreende três cartões com 2001 seis fileiras de cinco digitos unitários por fileira e os alunos são solicitados a dizer os números o mais rapidamente possível, iniciando pela coluna superior e procedendo até a última coluna. O tempo necessário para nomear os dígitos é marcado com um cronômetro.

Nomeação de letras: A atividade é semelhante à anterior, exceto pelo fato de que os estímulos são letras.

Nomeação de dígitos e letras: Neste caso, os estímulos são letras e números, seguindo o mesmo padrão das tarefas anteriores.

Geary, Hamson Repetição de três palavras monossilabas: (por exemplo,

\& Hoard (2000) school - tree - cake). O aluno lê a série de palavras até que possa memorizá-las e após repete a série, duas vezes seguidas, o mais rápido possível. Um cronômetro é utilizado para medir a velocidade de articulação das palavras.

Repetição de três pseudopalavras monossilabas: (por exemplo, lote, dake, pog). O procedimento da tarefa é idêntico ao anterior.

Repetição de três números de um dígito: (por exemplo, 2, $9,5)$. O procedimento da tarefa é idêntico ao anterior.

Bull \& Johnston Velocidade de contagem: A tarefa requer que o aluno conte

(1997) o número de pontos verdes presentes em cinco cartőes. Cada cartão contém entre um e nove pontos verdes e entre um e nove pontos vermelhos. É calculado o número de pontos contados por segundo.

Números Idênticos: A tarefa consiste de 30 fileiras de seis dígitos. O aluno deve identificar, o mais rápido possivel, dois dígitos idênticos em cada fileira. O tempo para completar as 30 fileiras é computado.

Figura geométrica idêntica: A tarefa consiste em identificar cinco figuras geométricas idênticas ao modelo apresentado (por exemplo, um triângulo com um ponto no centro) dentre 19 figuras alternativas (triângulos contendo diferentes objetos no seu centro, por exemplo, um quadrado, uma estrela, um sinal de mais). São apresentados 12 modelos de figuras geométricas. O tempo que o aluno leva para resolver toda a tarefa é cronometrado.

O estudo de Corso (2008) avaliou a velocidade de processamento em alunos da 3ạ a 6ạ série do Ensino Fundamental, com e sem dificuldades de aprendizagem na leitura e na matemática, por meio de três tarefas: nomeação de série de dígitos, série de letras e série de dígitos e letras (tarefa adaptada de Hecht et al., 2001). Os alunos sem dificuldades apresentaram o menor tempo (maior velocidade) 
para o processamento das três tarefas propostas. Já os alunos com dificuldades na leitura (DL) e na leitura e na matemática (DLM) mostraram desempenho inferior naquelas tarefas, necessitando de um tempo maior (menor velocidade) para o processamento. Um padrão similar de resultados foi encontrado por Geary, Hamson e Hoard (2000) ao observar que alunos com dificuldades na leitura e na matemática e alunos com dificuldades apenas na leitura apresentam uma velocidade na articulação de palavras conhecidas (medida de velocidade de processamento utilizada) mais baixa do que os alunos sem dificuldades de aprendizagem. O trabalho de Andersson (2008) também revelou que os alunos com dificuldades na leitura e na matemática apresentam problemas ligados à velocidade de processamento, já que estes realizaram a tarefa de identificar, o mais rápido possível, pares de números semelhantes (number matching task) de forma significativamente mais lenta do que os alunos sem dificuldades.

Algumas pesquisas sugerem que a velocidade de processamento é a habilidade cognitiva capaz de melhor predizer o desempenho em cálculo. O estudo de Bull e J ohnston (1997) incluiu alunos de primeira série, com e sem dificuldades na aritmética, que foram avaliados em uma série de medidas de velocidade para executar operações (velocidade de contagem, de identificação de números, de combinar números, de combinar formas geométricas, de desempenho percepto-motor, para fazer sequência e para realizar procedimentos aritméticos). O baixo desempenho dos alunos com dificuldades na matemática, em todas estas tarefas, indica que a velocidade de processamento representa um elemento de maior importância para explicar as dificuldades na aritmética.

Os resultados de Fuchs et al. (2008) apontam para a mesma direção. Os autores mostram que as dificuldades no cálculo estão associadas a uma baixa velocidade de processamento em alunos de 3 a série com dificuldades na matemática. O trabalho de Hecht et al. (2001) evidenciou que a velocidade de processamento foi um preditor significativo de diferenças em cálculo aritmético entre o 2 e e 30 ano de escolaridade, no entanto, o mesmo resultado não foi encontrado entre os alunos frequentando séries mais avançadas: 2 으 e 5으 ano, 3 은

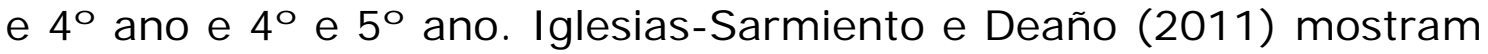
que a velocidade de processamento é um preditor específico de desempenho em matemática apenas no $1 \stackrel{0}{0}$ ano, mas o mesmo não ocorreu entre o 40 e o 60 ano.

O trabalho de Compton, Fuchs, Fuchs, Lambert e Hamlett (2012) não encontrou diferença na velocidade de processamento em alunos do 3o ao 5으 ano com e sem dificuldade em cálculo. Os autores procuraram explicar tais resultados baseados no fato de que a habilidade de cálculo para este nível de escolaridade torna-se mais complexa pela introdução de números racionais, o que pode exigir 
uma demanda cognitiva maior, diminuindo a velocidade de processamento até mesmo dos alunos sem dificuldades nesta área.

Os resultados de pesquisa apresentados por Cowan et al. (2011) replicam os achados de Fuchs et al. (2006) que encontraram um vínculo substancial entre a velocidade de processamento e o cálculo básico no 3o ano, mas não evidenciaram tal relação entre a velocidade de processamento e os problemas matemáticos.

Ao comparar os alunos com dificuldades persistentes na matemática (DM-p) e aqueles sem dificuldades, Geary, Hoard, Byrd-Craven, Nugent e Numtee (2007) observaram que aquele grupo executa medidas de nomeação rápida mais lentamente do que os colegas sem dificuldades no 1 을 ano, mas não no 2 으 ano. Da mesma forma, Murphy, Mazzocco, Hanich e Early (2007) evidenciaram que o grupo DM- $p$ realiza tarefas de nomeação rápida mais lentamente do que o grupo controle no jardim de infância e 1 ano, mas não no 2 으 ou 3 으 ano.

Os dados de pesquisa de Vucovic e Siegel (2010) não vão na mesma direção da hipótese de que déficits na nomeação rápida caracterizaria os alunos com dificuldades na matemática apenas nos anos escolares bem iniciais e não mais tarde. Os pesquisadores constataram que a tarefa de nomeação rápida foi capaz de distinguir os alunos com DM$\mathrm{p}$ dos sem dificuldades no 30 e no 4․ Do mesmo modo, Willcutt et al. (2013) evidenciaram que a velocidade de processamento pode exercer influência nas dificuldades de matemática de alunos mais velhos. Os autores observaram uma associação entre defasagens na velocidade de processamento e problemas na matemática, como também na leitura, em alunos com idades entre 8 e 15 anos.

Como vemos, não há consenso em muitos dos dados de pesquisa apresentados. As diferenças nos resultados dos estudos relacionando a velocidade de processamento e o desempenho em cálculo aritmético, em função dos diferentes níveis de escolaridade das amostras, ressalta a natureza desenvolvimental da velocidade de processamento, ou seja, a relação entre a velocidade de processamento e cálculo aritmético é mais pronunciada durante os primeiros estágios do desenvolvimento da aritmética e diminui à medida que as crianças se tornam mais proficientes. Berg (2008) destaca que a velocidade de processamento parece ter uma importância particular durante os primeiros estágios de desenvolvimento do cálculo aritmético (por exemplo, no desenvolvimento da automaticidade na representação de números na memória de longo prazo), e sua influência vai diminuindo com o tempo devido a um aumento da influência de outros fatores cognitivos (memória de curto prazo e memória de trabalho) e devido à sofisticação do desenvolvimento aritmético (aritmética simples progredindo para a aritmética mais complexa). 
Há, portanto, evidências que indicam que o desempenho mais lento, em especial em cálculo básico, é característica de alunos com dificuldades na matemática (Geary et al., 1991, Jordan \& Montani, 1997). Mas, de fato, não sabemos ao certo em que medida este processamento mais lento reflete características de um domínio cognitivo mais geral ou defasagens específicas na velocidade de processamento. Enquanto alguns estudos apontam a velocidade de processamento como um preditor independente de desempenho em cálculo (Bull \& Johnston, 1997), outros sugerem que a velocidade de processamento parece ter uma influência limitada na proficiência em cálculo (Andersson \& Lyxell, 2007, Jordan, Hanich \& Kaplan, 2003). Neste último caso, poderíamos pensar na influência que outra habilidade cognitiva subjacente à aprendizagem da matemática poderia exercer, como seria o caso da memória de trabalho, o que discutiremos na seção que segue.

\section{Relação entre velocidade de processamento e memória de trabalho}

Outra habilidade cognitiva frequentemente citada na literatura como prejudicada nos alunos com dificuldades na aritmética é a memória de trabalho: habilidade de armazenar e simultaneamente processar a informação.

Assim como a velocidade de processamento, estudos apontam que a memória de trabalho correlaciona-se com a proficiência em cálculo básico (Andersson \& Lyxell, 2007, Geary et al., 2007). Pesquisas mostram que tanto a velocidade de processamento quanto a memória de trabalho estão criticamente envolvidas com o desempenho em matemática, de tal forma que defasagens nestas áreas impedem o aluno de desenvolver habilidades matemáticas adequadas (Andersson \& Lyxell, 2007, Willcutt et al., 2013), como por exemplo, o desenvolvimento da fluência em fatos aritméticos, que vamos apresentar mais adiante.

Acredita-se que a velocidade de processamento interfere na capacidade de armazenar e recuperar informação numérica da memória de longo prazo, provavelmente através de uma ligação com a memória de trabalho (Vucovic \& Siegel, 2010). Grande parte da pesquisa em memória de trabalho é baseada no modelo de Baddeley e Hitch (1974). Segundo os autores, a memória de trabalho é formada por três componentes: o executivo central, o componente fonológico e o viso-espacial. O executivo central é o componente nuclear que possui capacidade de atenção limitada e é responsável pelo processamento de tarefas cognitivas. O componente fonológico e o e viso-espacial são subsistemas de armazenamento que têm 
capacidade limitada e estão em contato direto com o executivo central, sendo subordinados a ele e por ele recrutados.

A relação entre a velocidade de processamento e a memória de trabalho é bastante debatida na literatura. Existe um considerável consenso de que a memória de trabalho (com seus diferentes componentes) e a velocidade de processamento são fatores que contribuem para as dificuldades na matemática. Entretanto, existe inconsistência nos resultados quanto à relativa importância destes fatores (Bull \& Johnston, 1997, Mclean \& Hicht, 1999, Swanson \& Sachse-Lee, 2001). Discute-se se as variações individuais na memória de trabalho são ocasionadas por diferenças mais fundamentais na velocidade de processamento, ou se o foco atencional associado com 0 executivo central acelera 0 processamento da informação. Geary et al. (2007) destacam que, para os alunos com DM (amostra estabelecida a partir de um critério restritivo ${ }^{1}$ ), o componente executivo central da memória de trabalho mostrou ser um fator central nas dificuldades evidenciadas por este grupo em todas as tarefas de cognição matemática. Mas, ao mesmo tempo, os autores salientam que o componente fonológico, o visoespacial e a velocidade de processamento também podem contribuir para deficiências mais específicas na cognição matemática. Em outras palavras, diferentes componentes da memória de trabalho podem afetar diferentes aspectos do desempenho e da aprendizagem em matemática, o que pode explicar os resultados controversos presentes na literatura.

Em seu estudo, Berg (2008) investigou a contribuição da memória de trabalho e de outros processos cognitivos como a velocidade de processamento e a memória de curto prazo, para o desempenho em cálculo aritmético em alunos do 30 ao 60 ano. $O$ estudo evidenciou alguns achados interessantes que destacamos a seguir. A velocidade de processamento contribuiu significativamente para o cálculo aritmético, mas apenas para os alunos das séries mais iniciais da amostra, o que reforça o caráter desenvolvimental desta habilidade, como já mencionado anteriormente. Outra constatação foi a de que a velocidade de processamento e a memória de curto prazo não eliminaram a contribuição da memória de trabalho, como um todo, ou em seus componentes individuais, para o cálculo aritmético.

O autor lembra que embora a literatura argumente que tanto a velocidade de processamento como a memória de curto prazo parecem ser processos implícitos e necessários ao sistema de memória de trabalho, a questão que permanece é referente a que outros processos são responsáveis pela contribuição da memória de trabalho para o cálculo aritmético. Berg (2008) acredita que os recursos de atenção dentro da memória de trabalho (por exemplo, funções executivas relacionadas com a coordenação mental e flexibilidade) são caminhos prováveis para a interpretação destes 
resultados. O modelo revisado de Baddeley (2001) propôs que o executivo central não tem capacidade de armazenamento; ao contrário, ele é essencialmente um componente de processamento voltado para a atenção. Assim, o componente executivo central foca em processos de atenção diferenciados relacionados à atenção seletiva, sustentada e dividida. E, portanto, durante o cálculo aritmético, um processo de atenção específico ou uma combinação destes podem interagir. Pesquisas deste tipo podem trazer benefícios para a prática pedagógica que são inquestionáveis. Por exemplo, ao ensinar cálculo aritmético, os educadores poderiam desenvolver abordagens curriculares e metodológicas baseadas nos processos de memória que as crianças são susceptíveis a utilizar para resolver problemas aritméticos.

Finalmente, outro dado encontrado pelo autor foi que a memória de trabalho verbal e a memória de trabalho viso-espacial contribuíram, cada uma, como uma variância única para o cálculo na presença de todas as outras variáveis. Com tal resultado, Berg (2008) destaca a necessidade das pesquisas investigarem, com mais cuidado, os diferentes papeis potenciais do componente fonológico e viso-espacial para o cálculo aritmético, principalmente em crianças, considerando que ainda não está claro o papel preciso de cada componente.

Sem dúvida, é intenso e inconclusivo o debate em torno da relação entre a memória de trabalho e a velocidade de processamento. Enquanto alguns estudos definem a velocidade de processamento como um melhor preditor de desempenho em cálculo do que a memória de trabalho (Bull \& Johnston, 1997), outros referem a importante influência de ambos os processos (Willcutt et al., 2013). Ainda, outros estudos questionam se as diferenças individuais em memória de trabalho são influenciadas por diferenças mais fundamentais na velocidade de processamento ou se o foco de atenção associado com o executivo central acelera o processamento da informação (Engle, Tuholski, Laughlin \& Conway, 1999). Por ora, qualquer que seja a direção desta relação, concordamos com Geary (2011) quando ressalta que, ao nos propormos a avaliar os mecanismos potenciais que contribuem para as diferenças individuais no desempenho em cálculo, faz-se necessário a avaliação de ambos os processos cognitivos.

É importante considerarmos também em que medida os achados, bastante controversos nesta área, podem estar relacionados à questões metodológicas, do tipo o estabelecimento do ponto de corte utilizado pelas diferentes pesquisas para compor as amostras de alunos com dificuldades de aprendizagem na matemática. Alguns estudiosos, ao selecionarem suas amostras, utilizam pontos de corte mais restritivos (abaixo do percentil 10), enquanto outros se baseiam em pontos de corte mais lenientes (abaixo do percentil 30). Assim como lembram Murphy et al. (2007) e Geary et al. (2007), pontos de 
corte mais lenientes ou mais restritivos produzem amostras de sujeitos com capacidade distinta de memória de trabalho e velocidade de processamento. Ou seja, existe uma diferença qualitativa nas características dos grupos de alunos com DM em função do ponto de corte em questão. O uso de critérios mais restritivos identifica crianças com deficiências cognitivas abrangentes e severas e déficits subjacentes na memória de trabalho e velocidade de processamento. O uso de critérios mais lenientes identifica crianças que podem ter dificuldades mais sutis em alguns domínios da matemática. Os autores sugerem que as características dos alunos com dificuldades severas na matemática e com baixo desempenho diferem em aspectos importantes e que, por isso, estes grupos não podem ser fundidos.

\section{Velocidade de processamento e a fluência em fatos aritméticos}

A dificuldade para recuperar fatos numéricos básicos da memória de longo prazo é frequentemente citada na literatura como uma das características mais comuns, do mesmo modo que é a habilidade mais prejudicada nos alunos com dificuldades na matemática (Geary et al., 1999, 2000, Geary \& Hoard, 2001, Robinson, Menchetti \& Torgesen, 2002, Orrantia, Martinez, Morán, \& Fernández, 2002). Tal dificuldade se expressa através da permanência desses alunos no uso de estratégias de contagem mais primitivas e de uma lentidão para chegar a alcançar a recuperação de fatos da memória (Jordan \& Montani, 1997).

A velocidade de processamento dita o quão rápido os números podem ser contados. Portanto, ela pode facilitar a velocidade de contagem de forma que à medida que o aluno ganha velocidade na contagem de conjuntos para descobrir as somas e diferenças, os problemas são sucessivamente associados às suas respostas na memória de trabalho, antes de se perderem, de forma que aquela associação pode ser estabelecida na memória de longo prazo (Geary et al., 1991, Bull \& Jonhston, 1997). Por outro lado, um processamento mais lento aumenta o intervalo para derivar as associações problema-resposta na memória de trabalho. Isto cria a possibilidade de que o esquecimento ocorra antes mesmo que a sequência de cálculo seja completada. $O$ fortalecimento da associação de problemas-respostas na memória de trabalho requer que o tempo entre a decodificação do problema e a execução da resposta não ultrapasse o espaço 2 a 3 segundos (tempo que a memória de trabalho dispõe). Ao realiza um cálculo aritmético, o aluno pode utilizar um procedimento de contagem que requer um tempo superior ao que a memória de trabalho comporta, por exemplo, contar a partir 
da parcela maior (conta “5 $5,6,7,8$ " para resolver $3+5$ ). Neste caso, o aluno pode vir a ativar a resposta correta na memória de trabalho, mas a memória para o problema inicial $(3+5)$, provavelmente, se perderá, o que tornará inviável a associação fluente problema-resposta na memória de trabalho (Geary, 1993, Orrantia et al., 2002).

Vemos então que a velocidade de processamento está subjacente à fluência em fatos aritméticos e, por isso, autores como Hopkins e Lawson (2006) identificam tal habilidade como um determinante crítico do desenvolvimento da recuperação de fatos da memória. Descrevemos a seguir a pesquisa realizada por estes autores.

Hopkins e Lawson (2006) propõem que a velocidade de contagem, parte da velocidade de processamento, é um fator crítico que pode explicar por que alguns alunos não desenvolvem confiança na recuperação da memória apesar de utilizarem de forma eficiente a estratégia de contar a partir do maior, que naturalmente os levaria à decomposição e, após, à recuperação da memória.

Os autores destacam que o processo de desenvolver confiança na recuperação é mais sofisticado do que simplesmente um processo de memorização. Este requer que o aluno assimile o conhecimento de número para construir gradativamente estratégias backup (qualquer estratégia utilizada que não seja a de recuperação de fatos da memória) mais eficientes até que a prática continuada e eficiente com o uso destas estratégias fortaleça as associações problemaresposta para tornar a recuperação possível e provável.

Assim, para os alunos que apresentam um desenvolvimento típico, as estratégias de contagem menos eficientes (por exemplo, o contar nos dedos que demanda mais tempo e mais recursos da memória de trabalho) são substituídas por aquelas mais eficientes (por exemplo, estratégias de decomposição que permitem chegar mais rapidamente a resolução da situação problema: $6+7$ é solucionado recuperando a resposta para o problema $6+6$ e, então, adicionando 1 a esta soma parcial), construídas pelo aprendiz, eventualmente, levando-os ao desenvolvimento de associações problema-resposta que favorecem o desenvolvimento de confiança na recuperação.

Hopkins e Lawson (2006) observaram que um aspecto crítico que irá determinar se a prática usando a estratégia backup levará à confiança na recuperação é a velocidade de processamento, mais especificamente, o tempo levado pelo aluno para realizar a contagem ao executar a estratégia de contar a partir do maior. O trabalho também destaca que é pouco provável que a velocidade de contagem desempenhe, sozinha, um papel significativo. Os erros de contagem e as interrupções podem ser indicativos de dificuldades com 0 componente executivo central da memória de trabalho, responsável por inibir informação irrelevante. O fato dos alunos utilizarem os 
dedos para ajudá-los a manter a atenção em pequenos cálculos, sugere dificuldades com o componente executivo central.

Os resultados deste trabalho são muito importantes para a prática educacional. Uma baixa velocidade de contagem por si só não necessariamente constitui um déficit de aprendizagem, mas alunos com uma baixa velocidade de contagem não desenvolverão uma confiança na recuperação de fatos de adição considerando o mesmo ensino oferecido aos alunos com velocidades de contagem mais típicas.

Os autores argumentam que uma intervenção pedagógica de sucesso deve integrar de forma balanceada práticas que promovam o desenvolvimento do senso numérico e a confiança na recuperação. Neste ponto, enfatizam que desenvolver confiança na recuperação requer mais do que ensinar os alunos com DM estratégias de backup eficientes. Hopkins e Lawson (2006) se referem a práticas que promovam uma confiança na recuperação depois do aluno já utilizar em seu repertório estratégias backup de forma eficiente. Este último aspecto tem recebido pouca atenção na literatura e merece ser foco de pesquisa futura.

Um estudo piloto foi desenvolvido por Hopkins e De Villiers (2007) com 10 alunos australianos do 70 ano do Ensino Fundamental que não haviam desenvolvido confiança na recuperação de fatos básicos de adição simples. A intervenção focou o aumento da velocidade de contagem dos estudantes durante a realização da estratégia de contar a partir do maior e compreendeu um período de 5 sessões. Os resultados indicaram que a intervenção foi eficiente no aumento da média da velocidade de contagem dos alunos, aumentando assim a probabilidade de uso da estratégia de recuperação da memória.

Finalmente, destacamos nesta seção que a velocidade de processamento exerce um papel importante para a evolução e amadurecimento da estratégia de recuperação de fatos da memória de longo prazo. Tal estratégia, por sua vez, possibilita que o aluno adquira um nível de automatismo que lhe permitirá proficiência na matemática de forma que a execução de um cálculo, por exemplo, seja rápida e acurada, com pouco ou nenhum monitoramento consciente, de modo que recursos da atenção podem ser alocados para outras tarefas ou processos cognitivos (Gersten, Jordan, \& Flojo, 2005).

\section{Conclusão}

O estudo das habilidades cognitivas subjacentes à aprendizagem da aritmética vem crescendo, sendo foco de atenção de pesquisadores nas áreas de educação matemática, psicologia cognitiva e neuropsicologia. Dentre as habilidades pesquisadas, a memória de 
trabalho e a velocidade de processamento tem sido as mais estudadas.

Este artigo deu ênfase à velocidade de processamento e procurou relacionar tal habilidade com a memória de trabalho. Como vimos, as pesquisas mostram grande divergência nos resultados encontrados 0 que evidencia a necessidade de continuarmos investigando a interface entre a velocidade de processamento e as dificuldades de aprendizagem na aritmética.

No entanto, por ser um campo de pesquisa ainda recente é possível que tal divergência seja ocasionada por fatores tais como a falta de uniformidade nos instrumentos que avaliam velocidade de processamento e memória de trabalho, amostras de alunos com dificuldades na aritmética baseadas em pontos de corte distintos (mais lenientes ou mais restritivos) o que dificulta a generalização dos resultados, falta de padronização dos instrumentos que avaliam competência em matemática. Como consequência daqueles fatores, encontramos nos estudos grupos diferentes de crianças atendendo 0 critério para dificuldades na aritmética, em função dos instrumentos usados para a identificação das amostras e dos pontos de corte utilizados pelas pesquisas.

É fato que as pesquisas ainda não alcançaram um nível de especificidade que nos permita verificar em quais circunstâncias determinadas habilidades, tais como velocidade de processamento e memória de trabalho, atuam de forma independente, como preditores independentes de desempenho em aritmética, ou atuam em conjunto. De acordo com as pesquisas apresentadas, parece compreensível que as características da memória de trabalho possam afetar o desempenho em tarefas de velocidade de processamento, assim como a velocidade de processamento pode afetar 0 desempenho em tarefas de memória de trabalho, em uma perspectiva de complementariedade.

Para finalizar, não resta dúvida da necessidade de mais pesquisa que defina melhor o papel da velocidade de processamento e também da memória de trabalho na aprendizagem da matemática. Precisamos de evidências relacionando as intervenções naquelas áreas e 0 desempenho em cálculo. Pesquisas deste tipo trarão ricas contribuições para o desenvolvimento da intervenção preventiva (área que merece mais atenção) e corretiva das dificuldades de aprendizagem na aritmética. É fundamental conhecermos as habilidades cognitivas que estão prejudicadas nos alunos com dificuldades de aprendizagem, pois a forma como se conduz o ensino irá influenciar diretamente no efeito que o déficit cognitivo exercerá no aprender. Por exemplo, a aprendizagem da multiplicação poderá ser difícil para o aluno que enfrenta dificuldades para recuperar fatos da memória, se o ensino for baseado puramente na memorização da tabuada. Mas, ao contrário, se o professor focar na compreensão dos 
fatos básicos e ensinar estratégias de ação nas quais o aluno possa se basear para compensar sua dificuldade de memória, então aprender a multiplicação não será um grande problema para aquele aluno. Ou ainda, um aluno que se mostra demasiadamente lento para calcular, precisará do ensino de estratégias e procedimentos de contagem mais maduros e eficientes para que não necessite apoiarse demasiadamente na memória de trabalho (como ocorre quando é utilizado o procedimento de contar todas as parcelas da operação), sobrecarregando-a e aumentando a chance de erro no cálculo.

\section{Referências}

Andersson, U. (2008). Mathematical competencies in children with different types of learning disabilities. Journal of Educational Psychology, 100(1), 48-66.

Andersson, U., \& Lyxell, B. (2007). Working memory deficit in children with mathematical difficulties: A general or specific deficit? Journal of Experimental Child Psychology, 96(3), 197228.

Baddeley, A. D. (2001). Is working memory still working? American Psychologist, 56, 851-864.

Baddeley, A. D., \& Hitch, G. J. (1974). Working memory. In: G. H Bower (Ed.). The psychology of learning and motivation. (Vol. 8, pp. 47-91) London: Academic Press.

Berg, D. (2008). Working memory and arithmetic calculation in children: The contributory roles of processing speed, short-term memory, and reading. Journal of Experimental Child Psychology, 99, 288-308.

Bull, R., \& Johnston, R. S. (1997). Children's arithmetical difficulties: Contributions from processing speed, item identification, and short-term memory. Journal of Experimental Child Psychology, 65, 1-24.

Compton, D., Fuchs, L., Fuchs, D., Lambert, W., \& Hamlett, C. (2012). The cognitive and academic profiles of reading and mathematics learning disabilities. Journal of Learning Disabilities, 45(1), 79-95.

Corso, L. V. (2008). Dificuldades na Leitura e na matemática: um estudo dos processos cognitivos em alunos da 3a a 6a série do Ensino Fundamental. Tese de Doutorado, Universidade Federal do Rio Grande do Sul, Porto Alegre, RS, Brasil.

Cowan, R., Donlan, C., Shepherd, D., Fletcher, R., Saxton, M., \& Hurry, J. (2011). Basic calculation proficiency and mathematics achievement in elementary school children. Journal of Educational Psychology, 103(4), 786-803. 
Engle, R. W., Tuholski, S. W., Laughlin, J. E., \& Conway, A. R. (1999). Working memory, short-term memory, and general fluid intelligence: A latent variable approach. Journal of Experimental Psychology, 128, 309-331.

Fuchs, L. S., Fuchs, D., Compton, D. L., Powell, S. R., Seethaler, P. M., Capizzi, A. M., \& Fletcher, J. M. (2006). The cognitive correlates of third-grade skill in arithmetic, algorithmic computation, and arithmetic word problems. Journal of Educational Psychology, 98, 29-43.

Fuchs, L. S., Fuchs, D., Stuebing, K., Fletcher, J. M., Hamlett, C. L., \& Lambert, W. (2008). Problem solving and computational skill: Are they shared or distinct aspects of mathematical cognition? Journal of Educational Psychology, 100(1), 30-47.

Geary, D. C. (1993). Mathematical Disabilities: cognitive, neuropsychological and genetic components. Psychological Bulletin, 114, 345-362.

Geary, D. C. (2011). Cognitive predictors of achievement growth in mathematics: A 5-year longitudinal study. Developmental Psychology, 47(6), 1539-1552.

Geary, D. C., Brown, S. C., \& Samaranayake, V. A. (1991). Cognitive addition: A short longitudinal study of strategy choice and speed-of-processing differences in normal and mathematically disabled children. Developmental Psychology, 27, 787-797.

Geary, D. C., Hord, M. K., \& Hamson, C. O. (1999). Numerical and arithmetical cognition: patterns of functions and deficits in children at risk for mathematical disability. Journal of Experimental Child Psychology, 74, 213-239.

Geary, D. C., Hamson, C. O., \& Hoard, M. K. (2000). Numerical and arithmetical cognition: a longitudinal study of process and concept deficits in children with learning disabilities. Journal of Experimental Child Psychology, 77, 236-263.

Geary, D. C., \& Hoard, M. K. (2001). Numerical and Arithmetical Deficits in Learning-Disabled Children: relation to dyscalculia and dyslexia. Aphasiology, 15(7), 635-647.

Geary, D. C., Hoard, M. K., Byrd-Craven, J., Nugent, L., \& Numtee, C. (2007). Cognitive mechanisms underlying achievement deficits in children with mathematical learning disabilities. Child Development, 78(4), 1343-1359.

Gersten, R., Jordan, N., \& Flojo, J. (2005). Early identification and interventions for students with mathematics difficulties. Journal of Learning Disabilities, 38(4), 293-304.

Hecht, S., Torgesen, J., Wagner, R., \& Rashotte, C. A. (2001). The Relations between phonological processing abilities and emerging individual differences in mathematical computation skills: a longitudinal study from second to fifth grades. Journal of Experimental Child Psychology, 79, 192-227. 
Hopkins, S. L., \& Lawson, M. J. (2006). The effect counting speed has on developing a reliance on retrieval in basic addition. Contemporary Educational Psychology, 31, 208-227.

Hopkins, S., \& De Villiers, C. (2007). Bridging the gap between working memory research and teaching approaches in mathematics education. In: 12th European Conference for Research on Learning and Instruction (August 28 - September 1, 2007: Budapeste). Developing Potentials for Learning. [Abstracts]. Budapeste: Csapó; Csíkos.

Iglesias-Sarmiento, V., \& Deaño, M. (2011). Cognitive processing and mathematical achievement: A Study with schoolchildren between fourth and sixth grade of primary education. Journal of Learning Disabilities, 44(6), 570-583.

Jordan, N. \& Montani, T. (1997). Cognitive arithmetic and problem solving: a comparison of children with specific and general mathematics difficulties. Journal of Learning Disabilities, 30(6), 624-634.

Jordan, N., Hanich, L., \& Kaplan, D. (2003). A longitudinal study of mathematical competencies in children with specific mathematics difficulties versus children with comorbid mathematics and reading difficulties. Child Development, 74(3), 834-850.

Mclean, J. F., \& Hitch, G. J. (1999). Working memory impairments in children with specific arithmetic learning difficulties. Journal of Experimental Child Psychology, 74, 240-260.

Murphy, M. M., Mazzocco, M. M. M., Hanich, L. B., \& Early, M. C. (2007). Cognitive characteristics of children with mathematics learning disability (MLD) vary as a function of the cutoff criterion used to define MLD. Journal of Learning Disabilities, 40(5), 458-478.

Orrantia, J., Martinez, J., Morán, M., \& Fernández, J. (2002). Dificultades en el Aprendizaje de la Aritmética: Un analisis desde los modelos cronométricos. Cognitiva, 14(2), 183-201.

Robinson, C., Menchetti, B. M. \& Torgesen, J. (2002). Toward a twofactor theory of one type of Mathematics Disabilities. Learning Disabilities Research \& Practice, 17(2), 81-89.

Sasanguie, D., Van Den Bussche, E., \& Reynvoet, B. (2012). Predictors for mathematics achievement? Evidence from a longitudinal study. Mind, Brain, and Education, 6(3), 119-128.

Swanson, H. L., \& Sachse-Lee, C. (2001). Mathematical problem solving and working memory in children with learning disabilities: both executive and phonological processing are important. Journal of Experimental Child Psychology, 79(3), 294-321.

Vanbinst, K., Ghesquière, P., \& De Smedt, B. (2012). Numerical representations and individual differences in children's 
arithmetic strategy use. Mind, Brain and Education, 6(3), 129136.

Vukovic, R. K., \& Siegel, L. S. (2010). Academic and cognitive characteristics of persistent mathematics difficulty from first through fourth grade. Learning Disabilities Research and Practice, 25(1), 25-38.

Willcutt, E. G., Petrill, S. A., Wu, S., Boada, R., Defries, J., Olson, R., \& Pennington, B. (2013). Comorbidity between reading disability and math disability: concurrent psychopathology, functional impairment, and neuropsychological functioning. Journal of Learning Disabilities, 46(6), 500-516.

\section{Endereço para correspondência \\ Luciana Vellinho Corso}

Universidade Federal do Rio Grande do Sul

Av. Paulo Gama, 110 Prédio 12201 sala 929, Farroupilha, CEP 90040-060, Porto Alegre - RS, Brasil

Endereço eletrônico: I.corso@terra.com.br

\section{Beatriz Vargas Dorneles}

Universidade Federal do Rio Grande do Sul

Av. Paulo Gama, 110 Prédio 12201 70 andar, Farroupilha, CEP 90040-060, Porto Alegre - RS, Brasil

Endereço eletrônico: bvdornel@terra.com.br

Recebido em: 11/08/2014

Reformulado em: 03/10/2014

Aceito para publicação em: 06/10/2014

\section{Notas}

* Professora Adjunta do Departamento de Estudos Especializados da Faculdade de Educação da Universidade Federal do Rio Grande do Sul. Graduada em Pedagogia pela Universidade Federal do Rio Grande do Sul. Mestre em Educação pela Flinders University of South Australia. Doutora em Educação pela Universidade Federal do Rio Grande do Sul.

** Professora Associada do Programa de Pós Graduação em Educação da Faculdade de Educação da Universidade Federal do Rio Grande do Sul. Graduada em Pedagogia pela Universidade Federal do Rio Grande do Sul. Mestre em Educação pela Universidade Federal do Rio Grande do Sul. Doutora em Psicologia Escolar e do Desenvolvimento Humano pela Universidade de São Paulo. Pós-Doutorado pela Universidade de Oxford.

${ }^{1}$ O ponto de corte para os escores de desempenho em matemática utilizado nas pesquisas para definir a população de alunos com DM pode ser mais restritivos (< 5 th ou 10th percentil) ou mais lenientes (<30th percentil). O uso de critérios mais restritivos identifica crianças com deficiências cognitivas abrangentes e severas, já o uso de critérios mais lenientes identifica crianças que podem ter dificuldades mais sutis em alguns domínios da matemática. 